bioRxiv preprint doi: https://doi.org/10.1101/636845; this version posted May 14, 2019. The copyright holder for this preprint (which was not certified by peer review) is the author/funder, who has granted bioRxiv a license to display the preprint in perpetuity. It is made available under aCC-BY 4.0 International license.

\title{
Spatial Distribution of Cholesterol in Lipid Bilayers
}

M. Aghaaminiha, S. Sharma 


\section{Abstract}

We have performed molecular simulations to study spatial distribution and orientation of cholesterol molecules within three symmetric lipid bilayer systems: two binary lipid mixtures, namely, DOPC/CHOL (1, 2-dioleoyl-sn-glycero-3-phosphocholine/cholesterol) and SM/CHOL (d20:1/20:0 sphingomyelin /Cholesterol), and a tertiary mixture of DOPC/SM/CHOL. We have studied the behavior of these bilayers at temperatures varying from $400 \mathrm{~K}$ to $210 \mathrm{~K}$ and cholesterol molar concentration from $0 \%$ to $60 \%$.We observe that the spatial distribution of cholesterol is strongly correlated with the phase of the bilayer. In the disordered phase, cholesterol molecules are predominantly present near the center of the bilayer. In the ordered phase, cholesterol molecules are mainly present in the leaflets. At the order - disorder transition temperature, the fraction of cholesterol molecules is equal in the two leaflets and the center. In the leaflets, cholesterol molecules are oriented parallel to the bilayer normal, while near the center, cholesterol molecules are randomly oriented. In agreement with previous experimental studies, we find that increasing the cholesterol concentration favors ordered phase of the bilayers. The preference of cholesterol molecules to be present in the leaflets in the ordered phase is attributed to their favorable hydrophobic interactions with the lipid tails.

Keywords: Lipid bilayer, Cholesterol, Spatial distribution, Orientation, Molecular Simulation

\section{Statement of Significance}

Cholesterol has an important role in governing the physical properties of lipid bilayers, including their structural integrity, phase behavior and permeability. The spatial distribution of cholesterol in lipid bilayers is not well-understood because of the challenges associated with performing experiments for such a measurement. We show, via molecular simulations, that the spatial distribution of cholesterol molecules is strongly correlated with the phase behavior of the lipid bilayers. In the ordered phase, cholesterol molecules are predominantly present in the leaflets, whereas in the disordered phase, cholesterol molecules are in the center region of the bilayer. These results are important for understanding the relationship between lipid bilayer composition and their biological function and response.

\section{Introduction}

Biological membranes act as boundaries to maintain internal organelles of cells safe from external environment (1-3). They form a semi-permeable interface that allows cells to interact with extracellular environment (2-5). In mammalian cells, biological membranes, also known as plasma membranes, are made up of proteins, lipids, sterols, and other molecules (1, 3). Proteins are thought to be responsible for the transport of molecules and for chemical signaling processes across plasma membranes $(3,6)$. The lateral mobility and function of membrane proteins is understood to be dictated by the structure of the membrane $(6,7)$. Cholesterols, the main sterol in plasma membranes, play an important role in the dynamics and structural properties of the membranes (1-3, 6, 7). Cholesterols affect bending modulus (8), and phase behavior of the membrane (9), and are important for the formation of rafts domains (10). In addition, cholesterols 
affect membrane fluidity, permeability (7, 11), lateral diffusivity (7), thickness, structural orientation $(2,7,8,10,12)$, and lateral density $(2,5,7)$ of lipids in the membrane. Since a lipid bilayer is the dominant constituent of plasma membranes, study of their behavior as a function of cholesterol concentration and temperature can provide useful insights about plasma membranes (5, 7, 9, 11, 13-15). However, within lipid bilayers, the spatial distribution of cholesterol has not yet been clearly established, mainly because of the difficulty associated with performing experiments for such a measurement (12).

Cholesterol solubility in a lipid bilayer is known to be governed by the size of head group of lipids as well as by lipids' packing density $(16,17)$. Feigenson and Buboltz in 2001 reported that the maximum cholesterol concentration in the ternary mixture of DLPC/DPPC/CHOL is 66\%, and above that, monohydrated crystals of cholesterol precipitate out from the bilayer (18). The head group of lipids acts as a shield to isolate cholesterols from the lipid-water interface $(16,18)$. Thus, in lipids with a larger head group, such as sphingomyelins, cholesterol has higher solubility. Furthermore, it is reported that cholesterol solubility in membranes composed of saturated lipids is significantly higher in comparison to in membranes with monounsaturated/unsaturated lipids $(12,16,19)$.

Lipid bilayers are known to predominantly display two phases: ordered (liquid or gel) and disordered. These phases can be distinguished by considering translational order and chain configurational order of lipids in the bilayers $(12,16)$. The translational order refers to local structure of lipids and can be studied by calculating two dimensional radial distribution function (2D RDF) of lipid molecules in the bilayer plane. Chain configurational order is a measure of alignment of hydrocarbons tails of lipids in the bilayer. In the ordered phase, both translational order and chain configurational order are high, while in the disordered phase, both these orders are low. In the binary and ternary mixtures, cholesterol is well-known to favor the formation of the ordered phase $((9,12,13,20)$, by enhancing the chain configurational order $(12,16,21)$.

Molecular simulations have been useful in improving our understanding of biological processes, including the behavior of cell membranes (2, 22). While all-atom MD simulations have been employed to study dynamics and structural properties of lipid bilayers, these simulations are limited to time-scales of $\mathrm{O}\left(10^{2} \mathrm{~ns}\right)$ and length-scales of $\mathrm{O}(10 \mathrm{~nm})(23,24)$. Study of equilibrium phase behavior of lipid bilayers requires time-scales of $\mathrm{O}(1 \mu \mathrm{s})(7)$. Coarse-grained (CG) models reduce degrees of freedom of a system, and thus allow us to study longer length- and time-scales $(23,25)$. MARTINI force field is a popular CG force field for the study of lipid bilayers, surfactants, cholesterol, sugars and proteins $(23,26,27)$. Along with the explicit solvent version (called Wet MARTINI), an implicit solvent version of the force field, known as Dry MARTINI has been recently developed (27). In the MARTINI force field, the coarse graining of molecules follows a four-to-one mapping, that is, each CG bead (or interaction site) represents four heavy atoms (23, 26). There are four main types of beads: polar, non-polar, apolar, and charged, along with several sub-types. In the Dry MARTINI force field, the non-bonded interactions matrix from wet MARTINI force field is re-parameterized to match the experimental values of hydration, vaporization, and partitioning free energies (27).

In this work, we have studied the spatial distribution and orientation of cholesterol molecules in DOPC/CHOL (1, 2-dioleoyl-sn-glycero-3-phosphocholine/cholesterol), SM/CHOL (d20:1/20:0 sphingomyelin /Cholesterol), and DOPC/SM/CHOL mixtures over a range of temperatures and cholesterol concentrations using CG MD simulations. Lipids and cholesterol molecules are modeled using the Dry MARTINI force field (see Figure 1). We have calculated structural and dynamical properties of the bilayers to identify different phases as a function of 
temperature and cholesterol concentration. We find that in the ordered phase, cholesterol is mainly present in bilayer leaflets, while in the disordered phase, cholesterol is predominantly present near the center of the bilayer. In the leaflets, cholesterol molecules are oriented parallel to the bilayer normal, whereas, cholesterol molecules near the center of the bilayer are randomly oriented with respect to the bilayer normal. Favorable hydrophobic interactions between the lipid tails and cholesterol molecules drives the preference of cholesterol to enter the leaflets.
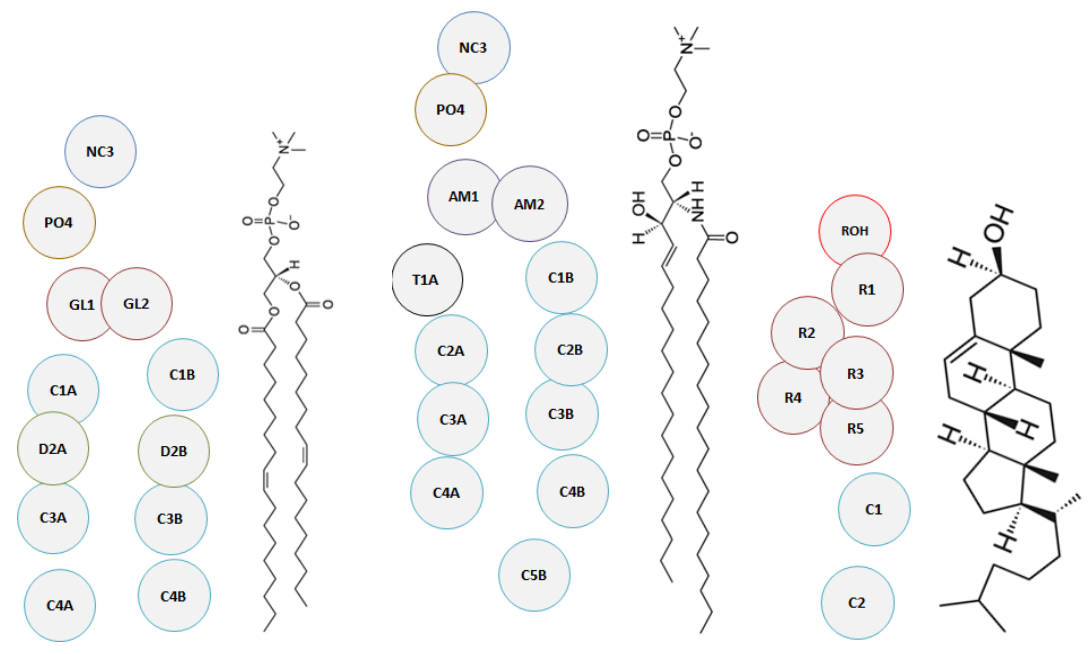

Figure 1: Dry Martini coarse grained beads. From left to right: DOPC, SM, and Cholesterol (28-30)

\section{Simulation System and Methods}

We studied three different types of lipid bilayers: DOPC/CHOL, SM/CHOL, and 1:1 DOPC/SM/CHOL. DOPC represents a lipid with low melting temperature. SM represents a lipid with relatively high value of melting temperature. All lipids and cholesterol molecules were modeled using Dry MARTINI version v2.0 force field. Periodic boundary conditions were applied in all three directions of the simulation box. All simulations were performed using the GROMACS/5.1.2 (31) molecular simulation package. The initial configuration of the three bilayer systems was constructed using the insane.py script developed by Marrink et al (28). The total number of molecules (lipids and cholesterols) were similar in all the simulations ( 512, kindly see Table S1, Supporting Information for the exact numbers). The concentration of cholesterol in the lipid bilayers was varied from $0 \%$ to $60 \%$ mole-ratio.

\subsection{Simulation Protocol}

After generating the initial configuration of the lipid bilayer, the energy of the configuration was minimized using the steepest descent algorithm. Next, to equilibrate the bilayer structure, a 50 ns NVT ensemble simulation followed by a 50 ns NPT ensemble simulation was performed at a constant temperature of $400 \mathrm{~K}$. The time-step for the simulations was $10 \mathrm{fs}$. After the simulation at $400 \mathrm{~K}$, the system was cooled to $390 \mathrm{~K}$ and a 10 ns equilibration followed by a $40 \mathrm{~ns}$ of production run was performed. This way, the entire temperature range from $400 \mathrm{~K}$ to 210 $\mathrm{K}$ was studied. For the three bilayer systems at temperatures $<300 \mathrm{~K}$, we performed $500 \mathrm{~ns}$ of production run to ensure invariability of results. For the three bilayers, we also studied systems with four times the number of molecules for few temperatures to ensure there are no finite size 
effects in the results. Figure 2 shows the initial configuration of DOPC/SM/CHOL system before performing MD production run at $400 \mathrm{~K}$.
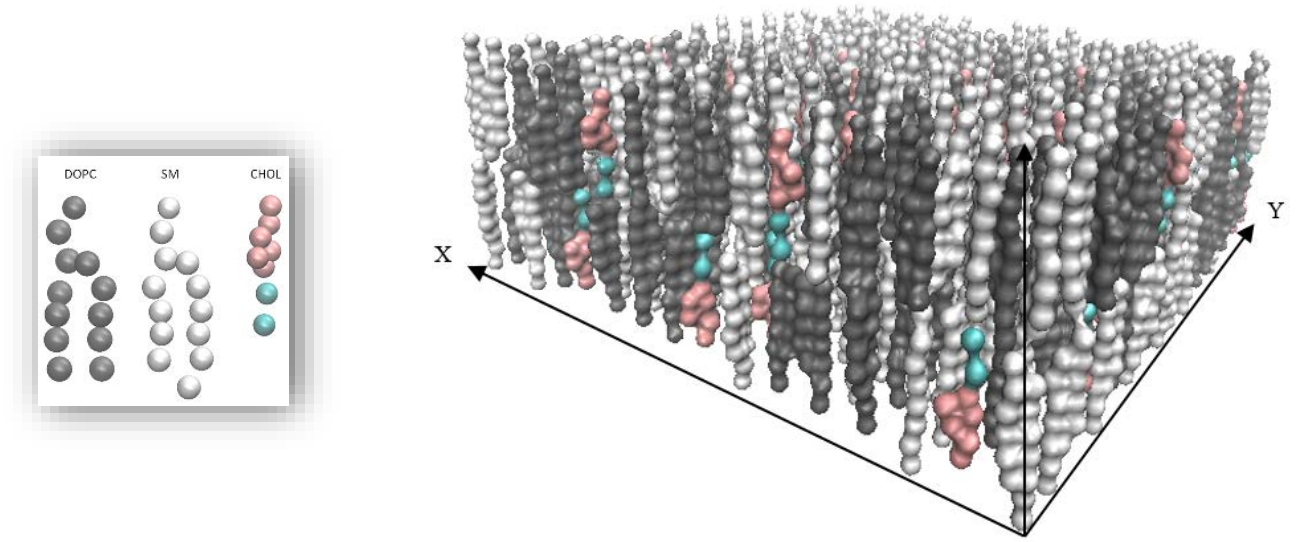

Figure 2: Mixture of DOPC/SM/CHOL initial configuration (at molar cholesterol concentration of 10\%)

\subsection{Simulation parameters}

Spherical cut-off for Lennard-Jones interactions was taken as $1.2 \mathrm{~nm}$. The non-bonded forces were smoothly switched to zero beyond the spherical cut-off. Velocity-rescaling thermostat developed by Bussi et al (32) was used as the temperature coupling method. The time constant for the thermostat was taken to be 1 ps. For the NPT runs, Parrinello-Rahman barostat was employed with the time constant of 4 ps. In the NPT runs, the membrane was made tensionless by setting the reference pressure to 0 bar in $x y$-plane. Also, the isothermal compressibility was set to $3 \times 10^{-4}$ bar $^{-1}$ in the $x y$-plane and $0 \mathrm{bar}^{-1}$ in $z$ direction (27). To remove net motion of the bilayer, the center of mass motion was subtracted out.

\subsection{Bilayer properties analysis}

Area per lipid was calculated as the ensemble-averaged area of the plane in the simulation box in which the bilayer resides divided by the number of lipids in one leaflet. Bilayer thickness was calculated as the ensemble-averaged distance between the $\mathrm{PO}_{4}$ beads (see Figure 1) of lipids in each leaflet. The average tail angle with bilayer normal was calculated as the angle between vector-a (vector connecting the first bead to the last bead of each tail, see Figure 1) and vector-b ( $\mathrm{z}$ axis). To monitor ordering of lipid chains we calculated the ensemble-averaged order parameter as follows:

$$
S_{\text {chain }}=\left\langle\frac{1}{2}\left(3\left\langle\cos ^{2} \theta\right\rangle-1\right)\right\rangle
$$

Where $\theta$ is the angle between each bond vector of the CG lipid beads and the bilayer normal (z-axis). High value of the $S_{\text {chain }}(\sim 1)$ indicates that the bonds are aligned with the bilayer normal, representing the chain order characteristic of the ordered phase $(10,16)$ while its low value $(\sim 0)$ indicates that the bonds are randomly oriented with respect to the bilayer normal, a signature of the disordered phase $(10,16)$. To quantify translational ordering of lipids, we calculated two dimensional (2D) radial distribution function (RDF). Long-ranged and more ordered correlations belong to the ordered phase while short-ranged correlations that decay rapidly represent the disordered phase. We calculated the 2D RDF based on the C1B beads (see Figure 1) of lipids, 
similar to (1) since it is located in the middle of both DOPC and SM and can be taken as the center of mass of the lipids. Phase behavior of lipid bilayers has been extensively studied before, and different phases have been classified as gel, liquid-ordered and liquid-disordered etc. Through heuristic rules to identify these phases, previous simulation studies have developed phase diagrams of lipid bilayers. In this work, our focus is to study spatial distribution of cholesterol in lipid bilayers as a function of the structure of lipid bilayers. Therefore, we have classified the behavior of lipid bilayers more broadly in terms of ordered and disordered phases only.

In addition to the structural properties mentioned above, we calculated the lateral diffusion coefficients of lipids in the bilayers. The angle of cholesterol molecules with the bilayer normal (z-axis) was also calculated as the ensemble-averaged of the angle between vector-a (vector connecting ROH bead to C2 bead, see Figure 1) and vector-b (z axis).

\section{Results and Discussion}

To determine spatial distribution of cholesterol molecules within the lipid bilayer and find its dependence on the phase of the bilayer, we studied three lipid/cholesterol mixtures, namely, DOPC/CHOL, SM/CHOL, and DOPC/SM/CHOL. For each system, we studied bilayer properties by varying the temperature from $400 \mathrm{~K}$ to $210 \mathrm{~K}$ in decrements of $10 \mathrm{~K}$ for seven different cholesterol concentrations ranging from $0 \%$ to $60 \%$.

5.1. Behavior of lipid bilayers as a function of temperature in the absence of cholesterol
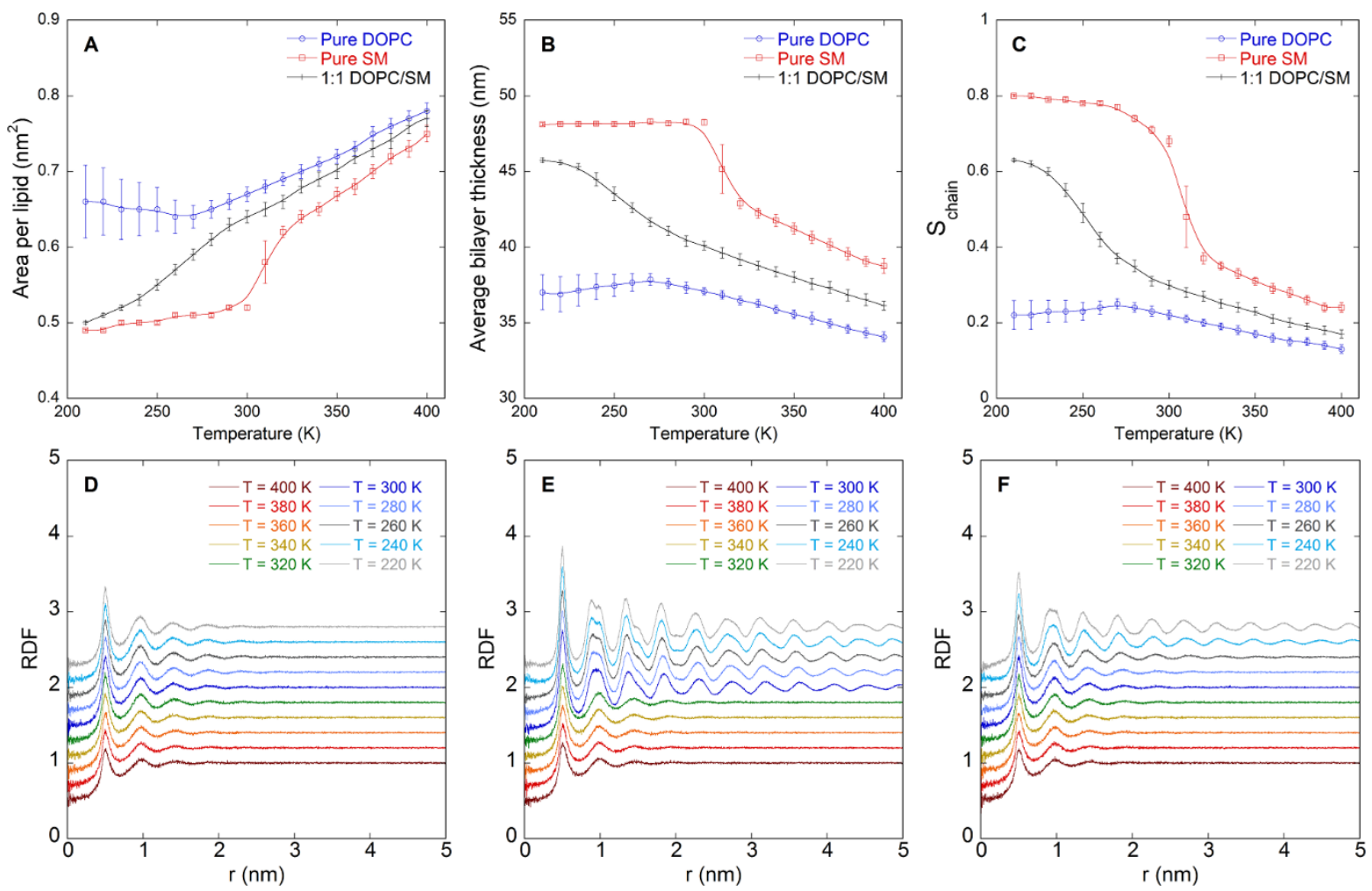

Figure 3: Bilayer structural properties as a function of temperature: Area per lipid (A), average bilayer thickness (B), average order parameter (Schain) $(C), 2 \mathrm{D}$ RDF of pure DOPC (D), pure SM (E), and 1:1 DOPC/SM (F).

Successive 2D RDFs have been shifted vertically by 0.2 units for the sake of clarity 
As a first step, we calculated structural properties of lipid bilayers of pure DOPC, pure SM and 1:1 mixture of DOPC/SM in the absence of any cholesterol. Figure 3 shows area per lipid, average bilayer thickness, average order parameter $\left(S_{\text {chain }}\right)$, and 2D RDF. Other structural and dynamical properties of bilayers such as average tail angle, total energy, and diffusivity are presented in Figure S1 (Supporting Information). From structural properties (Figure 3 A to C) as well as from 2D RDFs (Figure $3 \mathrm{D}$ to $\mathrm{F}$ ), it is observed that pure SM and 1:1 mixture of DOPC/SM have significant ordering of lipids at low temperatures, and as the temperature increases, they undergo an order - disorder transition. In contrast, pure DOPC bilayer does not show significant ordering even at the lowest temperatures sampled. This behavior of DOPC lipids matches with experimental observations as well (14). To estimate the transition temperature, $T_{m}$ of pure SM and 1:1 mixture of DOPC/SM systems, we fitted a polynomial function to the area per lipid graph and determined the location of the inflexion point by setting the second derivative to zero. Figure S2 (Supporting Information) shows plot of the derivative of the fitted polynomial. The $T_{m}$ of pure SM is estimated to be $315 \mathrm{~K}$, which agrees the experimentally reported value of $313 \mathrm{~K}$ (13). The $T_{m}$ of 1:1 mixture of DOPC/SM system is estimated to be $250 \mathrm{~K}$. Interestingly at the $T_{m}$ for both SM and DOPC/SM systems, $S_{\text {chain }} \sim 0.5$. Furthermore, the 2D RDFs presented in Figure 3 (E and F) do show loss of long-range structure as the temperature increases beyond the $T_{m}$. For DOPC, the $S_{\text {chain }}$ never goes above 0.3 , and the $2 \mathrm{D}$ RDFs do not show any long-range structure, supporting the conclusion that the DOPC lipid bilayer does not have ordered structure at any of the sampled temperatures.

\subsection{Spatial distribution of cholesterol in lipid bilayers}
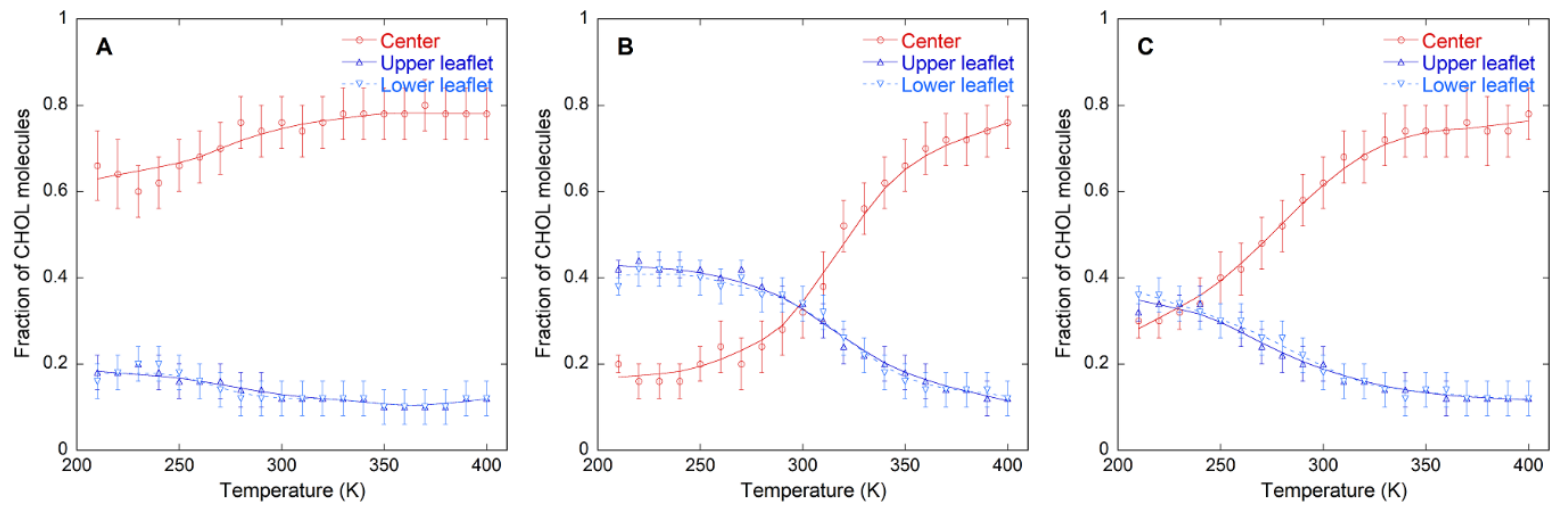

Figure 4: Fraction of cholesterol molecules at different regions of the bilayer (at molar cholesterol concentration of 10\%). Red, dark blue, and light blue lines represent cholesterol molecules located at the center, upper leaflet, and lower leaflet, respectively. DOPC/CHOL (A), SM/CHOL (B), and DOPC/SM/CHOL (C)

We performed molecular simulations of the three lipid bilayer systems with molar concentration of cholesterol varying from $10 \%$ to $60 \%$ using the simulation protocol discussed above. Figure 4 shows fraction of cholesterol molecules in the upper leaflet, lower leaflet and center of the lipid bilayers when the overall molar concentration of cholesterol is $10 \%$. The details of how upper leaflet, lower leaflet and center regions are defined are provided in Figure S3 (Supporting Information) and the accompanying text. From Figure 4, it is seen that in the case of DOPC, a larger fraction of cholesterol molecules are in the center region for all temperatures. However, for pure SM and 1:1 DOPC/SM mixture, there is higher fraction of cholesterol molecules in the upper and lower leaflets at lower temperatures. As temperature increases, the 
fraction of cholesterol molecules in the center region becomes higher. The crossover occurs close to the $T_{m}$ for these two lipid bilayer systems. Figure S4 (Supporting Information) shows the fraction of cholesterol molecules in the three lipid bilayer systems for molar cholesterol concentrations ranging from $20 \%$ to $60 \%$ as a function of temperature. It is observed that for all concentrations and temperatures, the cholesterol fraction is higher in the center region in the DOPC lipid bilayer. On the other hand, the pure SM and 1:1 DOPC/SM mixture show a crossover in the fraction of cholesterol molecules between the regions. The temperature at which the fraction of cholesterol molecules in the leaflet and the center regions becomes equal is denoted as $T_{\text {cross. }}$.

Figure 5 shows the $S_{\text {chain }}$ parameter for the three lipid bilayer systems for different cholesterol concentrations as a function of temperature. The order - disorder transition, $T_{m}$, for each system is denoted as the temperature at which $S_{\text {chain }}=0.5$. This definition of $T_{m}$ is employed because it is always found to be within a few Kelvins of the order - disorder transition temperature determined by locating the point of inflexion in structural properties of lipid bilayers (see Table S3, Supporting Information). Clearly from Figure 5A, the DOPC lipid bilayer is always in the disordered phase. For both SM and 1:1 DOPC/SM mixture, the $T_{m}$ shifts towards higher values as the concentration of cholesterol increases. The 2D RDFs of these systems are shown in Figures S6 (DOPC/CHOL), S7 (SM/CHOL) and S8 (DOPC/SM/CHOL) in Supporting Information. The shift in $T_{m}$ to higher values with increasing cholesterol concentration have also been reported for the system of DPPC/CHOL (7).
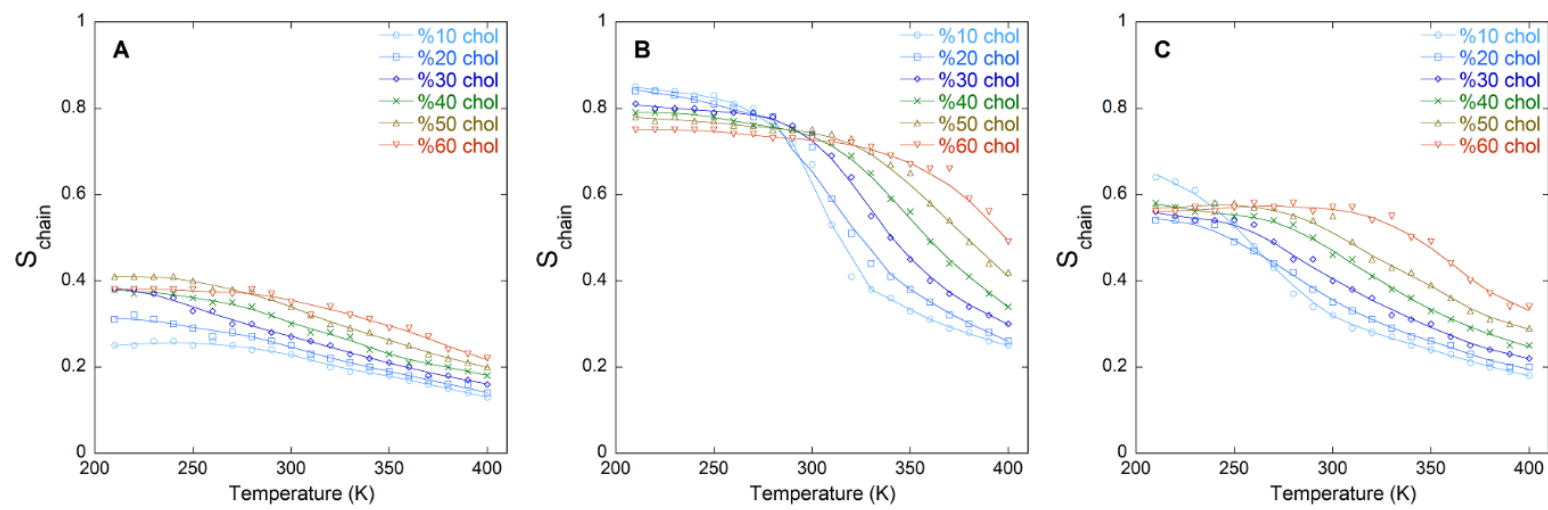

Figure 5: Average order parameter of chains $\left(S_{\text {chain }}\right)$ in the three systems: DOPC/CHOL (A), SM/CHOL (B), and $\mathrm{DOPC} / \mathrm{SM} / \mathrm{CHOL}(\mathrm{C})$

Figure 6A shows a plot of $T_{m}$ and $T_{\text {cross }}$ for the pure SM and DOPC/SM systems as a function of cholesterol concentration. Figure 6B shows a comparison of $T_{m}$ and $T_{c r o s s}$. The $T_{m}$ and $T_{\text {cross }}$ increase with cholesterol concentration, as expected. Also, the $T_{m}$ and $T_{\text {cross }}$ lie close to the $y$ $=x$ line in Figure 6B indicating that the spatial distribution of cholesterol is strongly correlated with the ordering in lipid bilayers.

According to Figure 4 and Figure S4 (Supporting Information), the DOPC/CHOL lipid bilayer system does not show any $T_{\text {cross }}$ and cholesterol always prefers to be in the center region. This matches with the observation that the DOPC/CHOL lipid bilayer is always in the disordered phase. The result that the DOPC system behaves differently from the other two systems is in agreement with the previous studies that have investigated the affinity of cholesterol in different types of lipids. In these studies, it is concluded that unsaturated fatty acids including lipids such as DOPC, have less affinity for cholesterol due to high disorder in these bilayers, however saturated lipids such as SM, have more favorable interactions with cholesterol $(12,13)$. 

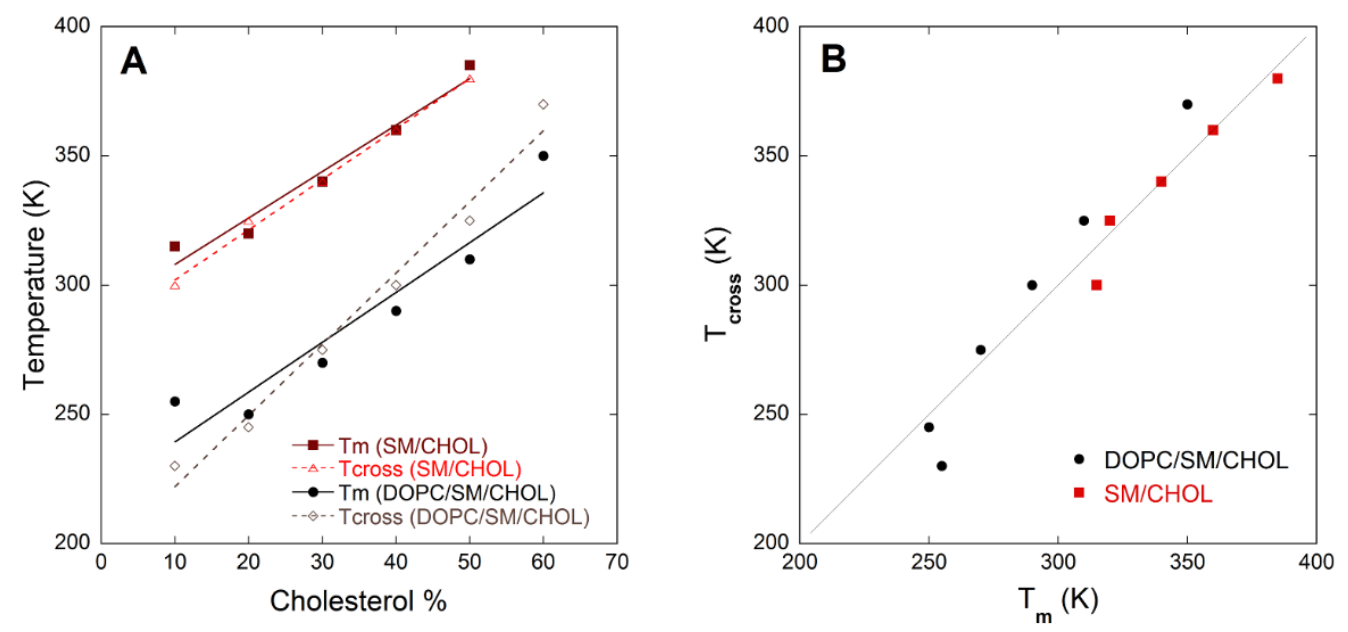

Figure 6: Spatial distribution of cholesterol vs phase represented with average order parameter $\left(\mathrm{S}_{\text {chain }}\right)$

Figure 7 shows density profiles of cholesterol molecules at different temperatures and for $10 \%$ molar cholesterol concentration for the three lipid bilayer systems studied. These profiles support our conclusion that cholesterol molecules prefer to be located at the center of the bilayer at high temperatures when the bilayer is in the disordered phase. At low temperatures for pure SM and 1:1 DOPC/SM mixture bilayers, cholesterol molecules prefer to be in the leaflets when the bilayer is in the ordered phase. Cholesterol is a hydrophobic molecule. In the ordered phase, it is able to form significant hydrophobic interactions with lipid tails. Hence, the entropic loss of entering the leaflets is compensated by the energetic gain of hydrophobic interactions. Figures S9 to S13 (Supplemental Information) display density profiles of the three systems at higher cholesterol concentrations (20\% to $60 \%)$. 

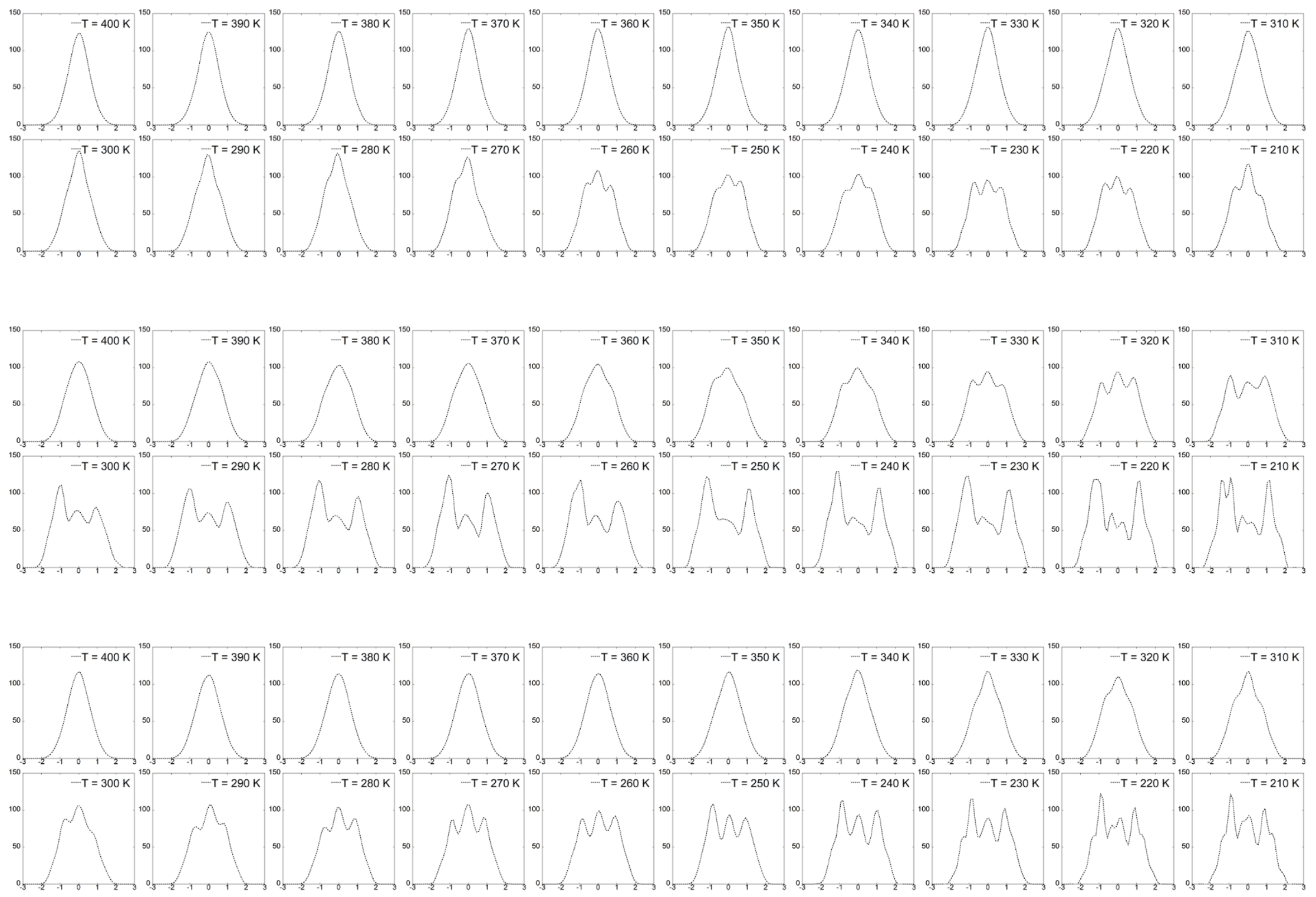

Figure 7: Cholesterol density profile at molar cholesterol concentration of 10\%. (Top) DOPC/CHOL, (middle) SM/CHOL, (bottom) DOPC/SM/CHOL In all plots, $y$ axis is the density of cholesterol in $g / L$ from 0 to 150; and $x$ axis is average relative position from center of the bilayer in $n m$ from -3 to +3 .

10 
bioRxiv preprint doi: https://doi. org/10.1101/636845; this version posted May 14 2019. The copyright holder for this preprint (which was not certified by peer review) is the author/funder, who has granted bioRxiv a license to display the preprint in perpetuity. It is made available under aCC-BY 4.0 International license.

Figure 8 shows snapshots of cholesterol molecules in the three lipid bilayers at $10 \%$ molar cholesterol concentration and at two temperatures $400 \mathrm{~K}$ and $210 \mathrm{~K}$. The snapshots show that SM/CHOL and DOPC/SM/CHOL lipid bilayers have ordered cholesterol molecules at $210 \mathrm{~K}$, while the DOPC/CHOL bilayer does not show any significant ordering.

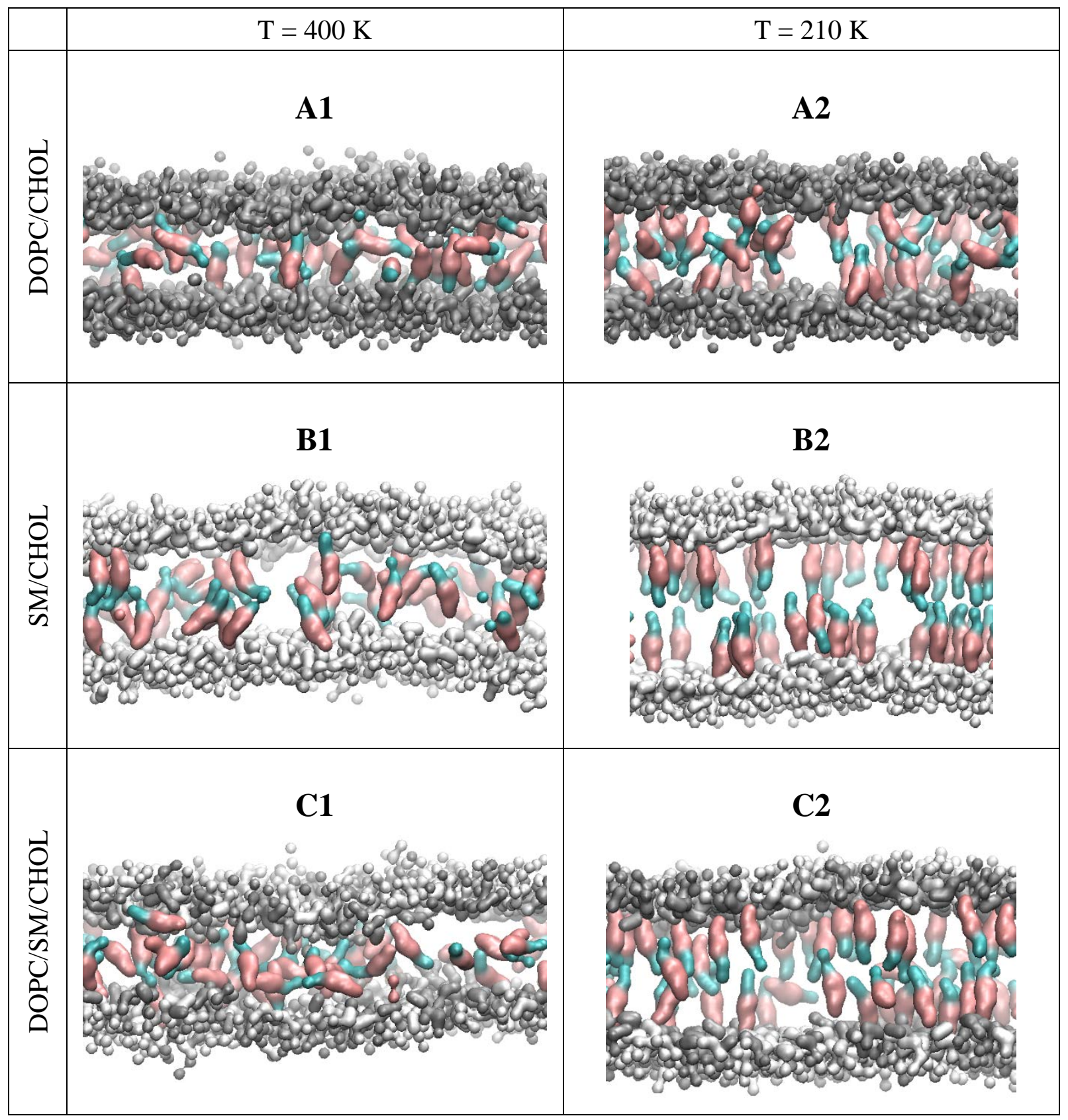

Figure 8: Simulations snapshots at $400 \mathrm{~K}$ and $210 \mathrm{~K}$ for the three systems (at molar cholesterol concentration of $10 \%$ ). The grey and white beads represent SM and DOPC head groups respectively, the maroon and cyan colors represent hydrocarbon rings and hydrocarbon tail of cholesterol, respectively. At $\mathrm{T}=400 \mathrm{~K}$ (Figure A1, B1, and C1), the thickness of the bilayers is small, cholesterol molecules are mainly at the center, and the orientation of cholesterol molecules is random. At $\mathrm{T}=210 \mathrm{~K}$ and for SM/CHOL and DOPC/SM/CHOL systems, (Figures B2 and C2), cholesterol molecules are oriented parallel to the bilayer normal. Tails of lipids are omitted in these snapshots for the sake of clarity. 


\subsection{Orientation of cholesterol molecules}

Figure 9 shows average angle that cholesterol molecules in the center region and leaflets make with the bilayer normal ( $z$ axis) when the molar cholesterol concentration is $10 \%$. In $\mathrm{SM} / \mathrm{CHOL}$ and DOPC/SM/CHOL bilayers, the cholesterol molecules are more aligned with the bilayer normal at low temperatures. The average angle in the leaflets is smaller than at the center, implying that cholesterol molecules align parallel to the bilayer normal in the leaflets. The average angle of cholesterol molecules in the three bilayers for higher cholesterol concentrations (20\% to 60\%) are shown in Figure S14 (Supporting Information). Figure 9 also shows that cholesterol molecules in the DOPC/CHOL system are less oriented with the bilayer normal as compared to the other two systems.
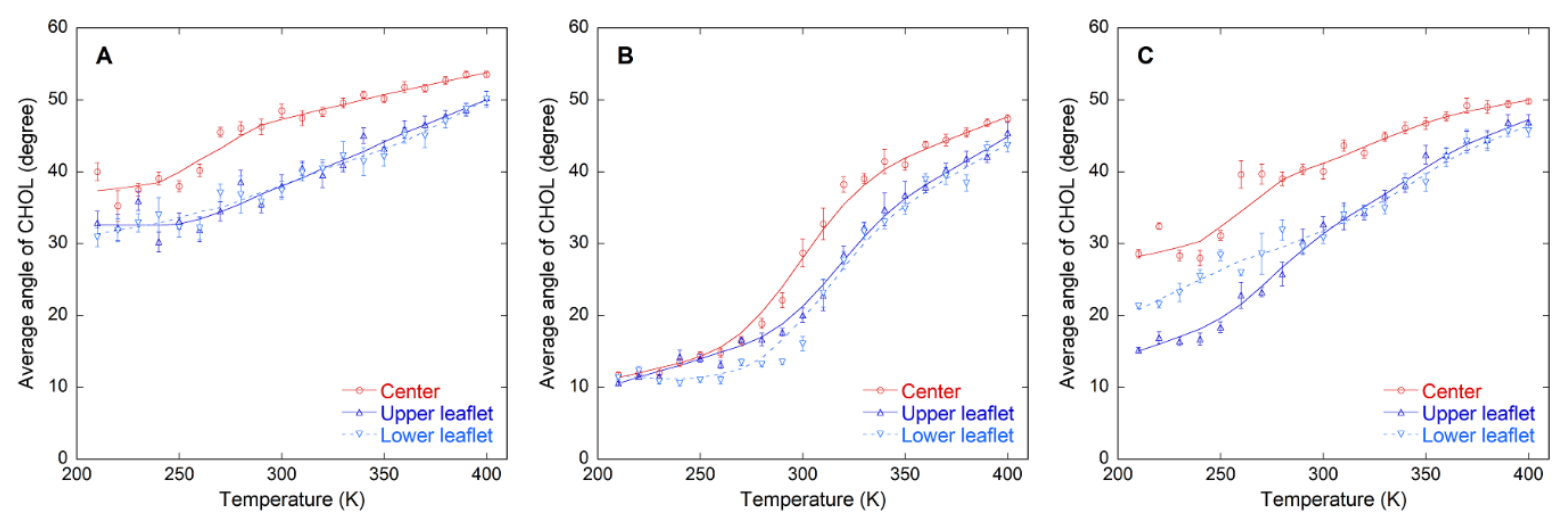

Figure 9: Orientation of cholesterol molecules in different regions of the bilayer (at molar cholesterol concentration of 10\%). Red, dark blue, and light blue lines represent cholesterol molecules located at the center, upper leaflet, and lower leaflet, respectively. DOPC/CHOL (A), SM/CHOL (B), and DOPC/SM/CHOL (C)

\section{Conclusions}

We have studied spatial distribution of cholesterol molecules as a function of cholesterol concentration and temperature in three lipid bilayer systems: DOPC/CHOL, SM/CHOL and $\mathrm{DOPC} / \mathrm{SM} / \mathrm{CHOL}$ using coarse-grained molecular simulations. We find that the spatial distribution of cholesterol molecules is closely related to the ordered and disordered phase of the bilayers. In the ordered phase, cholesterol molecules prefer to be in the leaflets and align themselves parallel to the surface normal. In this configuration, the cholesterol molecules maximize their hydrophobic interactions with the lipid tails. In the disordered phase, cholesterol molecules are preferentially in the center region of the bilayer. DOPC/CHOL bilayer does not form an ordered phase in the sampled temperature range. Therefore, for this system, the cholesterol molecules are always found predominantly in the center region. The temperature of the cross-over, $T_{\text {cross }}$ defined as the temperature at which the fraction of cholesterol molecules in the leaflets is equal to the fraction in the center region is found to be quite close to the order - disorder transition temperature, $T_{m}$ for all the bilayer systems studied.

\section{Authors Contribution}

MA and SS designed research. MA performed research. MA and SS performed analysis of simulation data. MA and SS wrote the manuscript. 


\section{Acknowledgements}

The authors would like to acknowledge Ohio University’s start-up funds for covering the computational costs of performing simulations at the Ohio Supercomputer Center. SS acknowledges additional computational resources from National Science Foundation XSEDE program (request number DMR190005).

\section{References}

1. Zhang Y, Lervik A, Seddon J, Bresme F (2015) A coarse-grained molecular dynamics investigation of the phase behavior of DPPC/cholesterol mixtures. Chem Phys Lipids 185:8898.

2. Zhuang X, Makover JR, Im W, Klauda JB (2014) A systematic molecular dynamics simulation study of temperature dependent bilayer structural properties. Biochim Biophys Acta BBA - Biomembr 1838(10):2520-2529.

3. Kučerka N, Nieh M-P, Katsaras J (2011) Fluid phase lipid areas and bilayer thicknesses of commonly used phosphatidylcholines as a function of temperature. Biochim Biophys Acta BBA - Biomembr 1808(11):2761-2771.

4. Gutknecht J, Walter A (1981) Hydrofluoric and nitric acid transport through lipid bilayer membranes. Biochim Biophys Acta BBA - Biomembr 644(1):153-156.

5. Khakbaz P, Klauda JB (2018) Investigation of phase transitions of saturated phosphocholine lipid bilayers via molecular dynamics simulations. Biochim Biophys Acta BBA - Biomembr 1860(8):1489-1501.

6. Lee AG (2004) How lipids affect the activities of integral membrane proteins. Biochim Biophys Acta BBA - Biomembr 1666(1):62-87.

7. Wang Y, Gkeka P, Fuchs JE, Liedl KR, Cournia Z (2016) DPPC-cholesterol phase diagram using coarse-grained Molecular Dynamics simulations. Biochim Biophys Acta BBA Biomembr 1858(11):2846-2857.

8. Pan J, Mills TT, Tristram-Nagle S, Nagle JF (2008) Cholesterol Perturbs Lipid Bilayers Nonuniversally. Phys Rev Lett 100(19):198103.

9. Feigenson GW (2009) Phase diagrams and lipid domains in multicomponent lipid bilayer mixtures. Biochim Biophys Acta BBA - Biomembr 1788(1):47-52.

10. Armstrong CL, et al. (2013) The Observation of Highly Ordered Domains in Membranes with Cholesterol. PLOS ONE 8(6):e66162.

11. Bagatolli L, Kumar PBS (2009) Phase behavior of multicomponent membranes: Experimental and computational techniques. Soft Matter 5(17):3234-3248.

12. Marquardt D, Kučerka N, Wassall SR, Harroun TA, Katsaras J (2016) Cholesterol's location in lipid bilayers. Chem Phys Lipids 199:17-25.

13. Konyakhina TM, Feigenson GW (2016) Phase diagram of a polyunsaturated lipid mixture: Brain sphingomyelin/1-stearoyl-2-docosahexaenoyl-sn-glycero-3phosphocholine/cholesterol. Biochim Biophys Acta BBA - Biomembr 1858(1):153-161.

14. Konyakhina TM, Wu J, Mastroianni JD, Heberle FA, Feigenson GW (2013) Phase diagram of a 4-component lipid mixture: DSPC/DOPC/POPC/chol. Biochim Biophys Acta BBA Biomembr 1828(9):2204-2214.

15. Ueda MJ, Ito T, Okada TS, Ohnishi SI (1976) A correlation between membrane fluidity and the critical temperature for cell adhesion. J Cell Biol 71(2):670-674. 
16. Marquardt D, Heberle FA, Nickels JD, Pabst G, Katsaras J (2015) On scattered waves and lipid domains: detecting membrane rafts with X-rays and neutrons. Soft Matter 11(47):90559072.

17. Buboltz JT, Feigenson GW (1999) A novel strategy for the preparation of liposomes: rapid solvent exchange. Biochim Biophys Acta BBA - Biomembr 1417(2):232-245.

18. Feigenson GW, Buboltz JT (2001) Ternary phase diagram of dipalmitoyl-PC/dilauroylPC/cholesterol: Nanoscopic domain formation driven by cholesterol. Biophys $J N Y$ 80(6):2775-88.

19. Harroun TA, Katsaras J, Wassall SR (2008) Cholesterol Is Found To Reside in the Center of a Polyunsaturated Lipid Membrane. Biochemistry 47(27):7090-7096.

20. Bennett WFD, MacCallum JL, Hinner MJ, Marrink SJ, Tieleman DP (2009) Molecular View of Cholesterol Flip-Flop and Chemical Potential in Different Membrane Environments. J Am Chem Soc 131(35):12714-12720.

21. Simons K, Toomre D (2000) Lipid rafts and signal transduction. Nat Rev Mol Cell Biol 1(1):31-39.

22. Berger O, Edholm O, Jähnig F (1997) Molecular dynamics simulations of a fluid bilayer of dipalmitoylphosphatidylcholine at full hydration, constant pressure, and constant temperature. Biophys J 72(5):2002-2013.

23. Marrink SJ, Risselada HJ, Yefimov S, Tieleman DP, de Vries AH (2007) The MARTINI Force Field: Coarse Grained Model for Biomolecular Simulations. J Phys Chem B 111(27):7812-7824.

24. Botan A, et al. (2015) Toward Atomistic Resolution Structure of Phosphatidylcholine Headgroup and Glycerol Backbone at Different Ambient Conditions. doi:10.1021/acs.jpcb.5b04878.

25. Bradley R, Radhakrishnan R (2013) Coarse-Grained Models for Protein-Cell Membrane Interactions. Polymers 5(3):890-936.

26. Marrink SJ (2016) Coarse Grain Forcefield for Biomolecules. Available at: http://cgmartini.nl/index.php/about [Accessed November 17, 2017].

27. Arnarez C ment, Uusitalo J (2014) Dry Martini, a Coarse-Grained Force Field for Lipid Membrane Simulations with Implicit Solvent. J Chem Theory Comput.

28. Wassenaar TA, Ingólfsson HI, Böckmann RA, Tieleman DP, Marrink SJ (2015) Computational lipidomics with insane: a versatile tool for generating custom membranes for molecular simulations. J Chem Theory Comput 11(5):2144-2155.

29. Melo MN, Ingólfsson HI, Marrink SJ (2015) Parameters for Martini sterols and hopanoids based on a virtual-site description. J Chem Phys 143(24):243152.

30. Marrink SJ, de Vries AH, Mark AE (2004) Coarse Grained Model for Semiquantitative Lipid Simulations. J Phys Chem B 108(2):750-760.

31. Abraham MJ, et al. (2015) GROMACS: High performance molecular simulations through multi-level parallelism from laptops to supercomputers. SoftwareX 1-2:19-25.

32. Bussi G, Donadio D, Parrinello M (2007) Canonical sampling through velocity-rescaling. $J$ Chem Phys 126(1):014101. 